\title{
A RELATION BETWEEN THE PERMANENTAL AND DETERMINANTAL ADJOINTS
}

\author{
MARVIN MARCUS' ${ }^{1}$ and RUSSELL MERRIS2
}

(Received 12 October 1970; revised 10 June 1971)

Communicated by G. Szekeres

Let $H_{n}$ denote the set of complex $n$-square positive semidefinite hermitian matrices. We partially order $H_{n}$ : If $A, B, A-B \in H_{n}$, write $A>B$. For $A \in H_{n}$, write $P(A)$ for the permanental adjoint of $A$, i.e., $P(A)$ is the $n$-square matrix whose $i, j$ entry is per $A(j \mid i)$, where $A(j \mid i)$ is the submatrix of $A$ obtained by deleting row $j$ and column $i$. Now, $P(A)$ is a principal submatrix of the $(n-1)$ st induced power matrix of $A^{T}$. Hence, $P(A) \in H_{n}$. Also $D(A)$, the classical adjoint, is in $H_{n}$.

THEOREM. If $A \in H_{n}$ is positive definite then

$$
(\operatorname{per} A)^{-1} P(A)<n(\operatorname{det} A)^{-1} D(A) .
$$

Proof. Rewrite (1) as follows:

$$
P(A)<n(\operatorname{per} A) A^{-1} .
$$

Pre- and post-multiply both sides of (2) by $A^{\frac{1}{2}}>0$ to obtain the equivalent statement

$$
A^{\frac{1}{2}} P(A) A^{\frac{1}{2}}<\mathrm{n}(\operatorname{per} A) I_{n} .
$$

Statement (3) is equivalent to the statement that the maximum eigenvalue of $A^{\frac{1}{2}} P(A) A^{\frac{1}{2}}$ satisfies

$$
\lambda_{1}\left(A^{\frac{1}{2}} P(A) A^{\frac{1}{2}}\right) \leqq n \text { per } A .
$$

Now, the eigenvalues of $A^{\frac{1}{2}} P(A) A^{\frac{1}{2}}$ are all nonnegative. Hence, it suffices to prove

1. The work of this author suuported was by the U. S. Air Force Office of Scientific Research under grant AFOSR 698-67.

2 The work of this author was done while he was a National Academy of SciencesNational Research Council Postdoctoral Research Associate at the National Bureau of Standards, Washington, D.C. 20234. 
that $\operatorname{tr}\left(A^{\frac{1}{2}} P(A) A^{\frac{1}{2}}\right) \leqq n$ per $A$. But, $\operatorname{tr}\left(A^{\frac{1}{2}} P(A) A^{\frac{1}{2}}\right)=\operatorname{tr}(A P(A))$. The main diagonal elements of $A P(A)$ are

$$
\sum_{k=1}^{n} a_{i k} \operatorname{per} A(i \mid k)=\operatorname{per} A .
$$

Hence, $\operatorname{tr}(A P(A))=n$ per $A$, and the proof is complete.

Indeed, the proof shows that (3) holds for all $A \in H_{n}$, not just for $A$ positive definite.

Corollary. Suppose $A \in H_{n}$. Let $\sigma_{i}$ be the ith row sum of $A$. Let $\sigma(A)$ be the sum of the elements of $A$. Then

$$
0 \leqq \sum_{i, j=1}^{n} \sigma_{i} \bar{\sigma}_{j} \operatorname{per} A(i \mid j) \leqq n \sigma(A) \operatorname{per} A .
$$

Proof. Display (3) is congruent to $A P(A) A<n(\operatorname{per} A) A$. Now, if $A \in H_{n}$ then $\sigma(A) \geqq 0$. It follows that

But

$$
0 \leqq \sigma(A P(A) A) \leqq n(\operatorname{per} A) \sigma(A) .
$$

$$
\sigma(A P(A) A)=\sum_{i, j=1}^{n} \sigma_{i} \bar{\sigma}_{j} \operatorname{per} A(i \mid j) .
$$

We point out that if $A \in H_{n}$ is doubly stochastic then the corollary becomes

$$
1 / n^{2} \sum_{i, j=1}^{n} \operatorname{per} A(i \mid j) \leqq \operatorname{per} A .
$$

Display (4) is the first of a class of inequalities conjectured by Djoković [1] to hold for all doubly stochastic $A$. It was proved in [2] using other methods.

\section{References}

[1] D. Ž. Djoković, 'On a conjecture by van der Waerden', Mat. Vesnik (4) 19 (1967), 272-276.

[2] Marvin Marcus and Henryk Minc, 'Extensions of classical matrix inequalities', Linear Algebra Appl. 1 (1968), 421-444.

The University of California

Santa Barbara

and

The National Bureau of Standards

Washington

U.S.A. 